\title{
REVIEW ARTICLE OPEN The importance of copy number variation in congenital heart disease
}

\author{
Gregory Costain ${ }^{1,2}$, Candice K Silversides ${ }^{3}$ and Anne S Bassett ${ }^{1,3,4,5}$
}

Congenital heart disease (CHD) is the most common class of major malformations in humans. The historical association with large chromosomal abnormalities foreshadowed the role of submicroscopic rare copy number variations (CNVs) as important genetic causes of CHD. Recent studies have provided robust evidence for these structural variants as genome-wide contributors to all forms of CHD, including CHD that appears isolated without extra-cardiac features. Overall, a CNV-related molecular diagnosis can be made in up to one in eight patients with CHD. These include de novo and inherited variants at established (chromosome 22q11.2), emerging (chromosome 1q21.1), and novel loci across the genome. Variable expression of rare CNVs provides support for the notion of a genetic spectrum of CHD that crosses traditional anatomic classification boundaries. Clinical genetic testing using genome-wide technologies (e.g., chromosomal microarray analysis) is increasingly employed in prenatal, paediatric and adult settings. CNV discoveries in CHD have translated to changes to clinical management, prognostication and genetic counselling. The convergence of findings at individual gene and at pathway levels is shedding light on the mechanisms that govern human cardiac morphogenesis. These clinical and research advances are helping to inform whole-genome sequencing, the next logical step in delineating the genetic architecture of CHD.

npj Genomic Medicine (2016) 1, 16031; doi:10.1038/npjgenmed.2016.31; published online 14 September 2016

Genome-wide rare copy number variation (CNV) is now recognised as an important contributor to congenital heart disease (CHD). This review surveys recent advances in the field of structural genomics, with the goal of informing both the clinical translation of findings and the anticipated future wave of whole-genome sequencing (WGS) studies. Herein, CHD refers to major malformations of the heart or great vessels present at birth. Cardiomyopathies, vasculopathies and cardiac arrhythmias are excluded. The focus is on findings from large-scale genome-wide studies of submicroscopic germline CNV, and not on chromosomal abnormalities detectable on karyotype, case reports or small case series of individual CNVs, or deletion/duplication analyses of individual genes.

\section{CLINICAL DESCRIPTION AND EPIDEMIOLOGY OF CHD}

CHD is the most common class of major congenital anomalies in humans, and a major source of morbidity and paediatric mortality around the world. ${ }^{1-3}$ Most incidence estimates range from 4 to 10 in 1,000 live births. ${ }^{1-3}$ Bicuspid aortic valve, isolated aneurysm of the atrial septum, and persistent left superior vena cava are usually excluded from these incidence figures. ${ }^{1}$ Although most cardiac defects can be diagnosed prenatally by fetal echocardiography, many anomalies are not identified on routine prenatal ultrasound. ${ }^{4-7}$ Prenatal diagnosis of severe CHD can improve outcomes. $^{5,7-9}$ Signs and symptoms are related to the type and severity of the heart defect. Many, but not all, cardiac lesions are identified in childhood. Surgical repair creates a new cardiac phenotype with unique associated late sequelae. Despite advances in early detection and surgical repair, CHD remains the leading global cause of non-infectious neonatal death. ${ }^{3,9}$ The vast majority of patients who survive this early period require lifelong specialised cardiac care. ${ }^{3,10-13}$

Beginning at 3 weeks of embryonic life, the heart is the first organ to start developing in the human embryo. ${ }^{14}$ An overview of the complex sequence of events that results in a well-formed heart at birth is beyond the scope of this review. ${ }^{14}$ Disruption of any developmental step can result in a cardiac malformation. Anatomical, functional and clinical categorisations exist for the myriad described cardiac lesions (Figure 1). Advances in imaging technology have led to improved diagnostic precision. ${ }^{7}$ Various constellations of multiple defects can be found together, and can result in more complex CHD, of which tetralogy of Fallot (TOF) is the most common form (Figure 1). ${ }^{11,12} \mathrm{CHD}$ may occur in apparent isolation, or in conjunction with other, extra-cardiac features (syndromic CHD). Overall, individuals with CHD are at increased risk for extra-cardiac congenital anomalies and for neurodevelopmental problems. ${ }^{15,16}$

With advances in paediatric surgery, the prevalence of individuals living with CHD is increasing in many areas of the world. ${ }^{2,3}$ Adults with CHD now outnumber affected children. ${ }^{17}$ With increased longevity, there is more attention on long-term outcomes and causation. Evidence from family and twin studies supports a genetic aetiology for CHD. ${ }^{18-21}$ However, classic

\footnotetext{
${ }^{1}$ Clinical Genetics Research Program, Centre for Addiction and Mental Health, Toronto, ON, Canada; ${ }^{2}$ Medical Genetics Residency Training Program, University of Toronto and

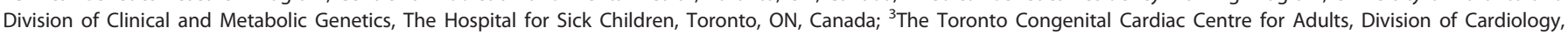
University Health Network and Mount Sinai Hospital, Toronto, ON, Canada; ${ }^{4}$ Department of Psychiatry, Toronto General Research Institute and The Dalglish Family Hearts and Minds Clinic for 22q11.2 Deletion Syndrome, University Health Network, Toronto, ON, Canada and ${ }^{5}$ Department of Psychiatry, University of Toronto, and Campbell Family Mental Health Research Institute, Centre for Addiction and Mental Health, Toronto, ON, Canada.
} 


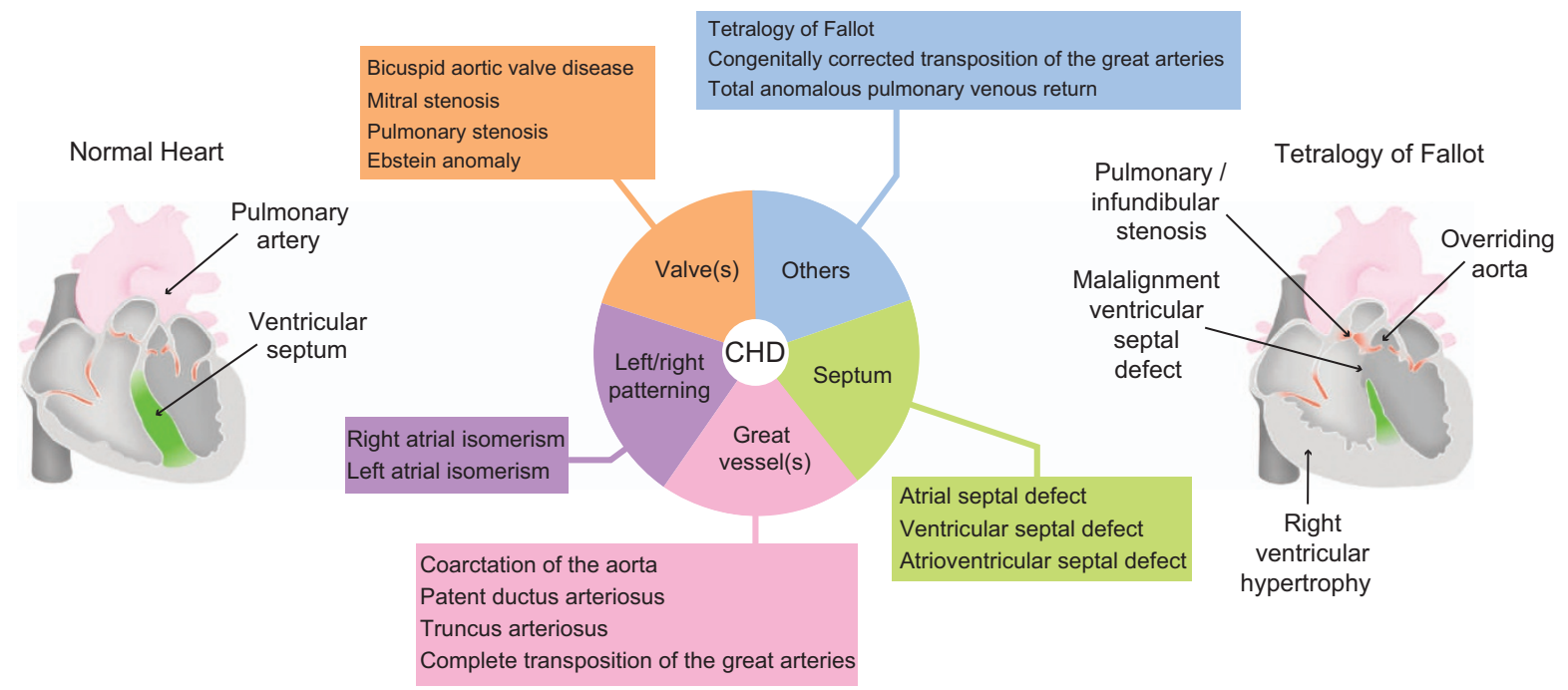

Figure 1. Spectrum of human CHD. CHD is an umbrella term for a range of malformations of the heart and great vessels (aorta and pulmonary arteries). There exist multiple clinically and anatomically discrete lesions, of differing incidence and severity. See text and associated references for details. (a) Labelled diagram of the structurally normal human heart. (b) Examples of some congenital cardiac lesions, based on anatomy. For a full list of congenital cardiac defects, consult a congenital cardiology textbook. Multiple congenital defects may be present within an individual. (c) Labelled diagram of one specific form of CHD: tetralogy of Fallot (TOF).

Mendelian inheritance patterns are usually not observed, reported recurrence risks are low, and there are low concordance rates of CHD within families. ${ }^{18-21}$ Non-genetic factors, e.g., teratogens such as alcohol or infections like rubella, or other comorbidities like maternal type 1 diabetes mellitus, may have a role in increasing risk for $\mathrm{CHD}$ and may interact with genetic predisposition. ${ }^{22,23}$ For these reasons CHD has long been considered an archetypal model for multifactorial inheritance..$^{20,24,25}$ Until recently, the main molecular genetic insights into the aetiology of CHD involved large chromosomal anomalies visible on karyotype.

\section{CONTEXT: EARLY STUDIES OF CHROMOSOMAL IMBALANCES IN CHD}

Major chromosomal anomalies including aneuploidies have been associated with CHD for over half a century. ${ }^{20}$ The major aneuploidies compatible with postnatal life are traditionally associated with certain types of CHD: Down syndrome/trisomy 21 (atrioventricular canal defect), Edward syndrome/trisomy 18 (ventricular septal defect or VSD, pulmonary stenosis), Patau syndrome/trisomy 13 (VSD, atrial septal defect, transposition of the great arteries or TGA), Turner syndrome/monosomy $X$ (coarctation of the aorta, aortic stenosis, VSD), and Klinefelter syndrome/XXY (Ebstein anomaly, TOF). ${ }^{26}$ It is important to note however that a broad spectrum of CHD has been observed within each of these syndromes.

Although genome-wide, the resolution of karyotype is low, rarely identifying structural anomalies less than $5-10 \mathrm{Mb}$ in size. Chromosomal abnormalities visible on karyotype often involve deletion or duplication of hundreds of genes, and expression of complex developmental phenotypes. ${ }^{20,27}$ Karyotype was thus not often helpful to identify individual genes important to cardiac morphogenesis. Over 20 years ago, a technological advance became clinically available that used targeted probe testing with fluorescence in situ hybridisation to provide a molecular diagnosis for selected microdeletion syndromes associated with CHD. ${ }^{20}$ Testing required a high clinical index of suspicion and knowledge of the targeted syndrome to allow the specific probe to be ordered.
Fluorescence in situ hybridisation was instrumental in revealing the presence of submicroscopic structural anomalies associated with identifiable genetic syndromes, e.g., 7p11.23 and 22q11.2 deletions, respectively, for Williams-Beuren syndrome and $22 q 11.2$ deletion syndrome (22q11.2DS), the latter formerly known as DiGeorge/velocardiofacial syndrome. ${ }^{28,29}$ Studies of these recurrent, usually de novo, $1.5-3 \mathrm{Mb}$ sized CNVs have proven useful to identify individual genes ( $E L N$ at the 7p11.23 locus and $T B X 1$ and $C R K L$ at the $22 q 11.2$ locus) that are important in cardiac morphogenesis. ${ }^{28,29}$ Animal models with genetically engineered deletions and point mutations of selected genes have been instrumental in these studies. ${ }^{28-30}$

These early findings have left the impression that most chromosomal anomalies associated with CHD: (i) arise as de novo mutations; (ii) usually result in multiple congenital extra-cardiac features making them readily identifiable in the newborn period; (iii) have a rather specific pattern of associated CHD per individual syndrome (e.g., supravalvular aortic stenosis in Williams-Beuren syndrome); and (iv) individually account for relatively few patients with CHD. It would take new molecular technologies to reveal otherwise.

\section{GENOME-WIDE CNV IN HEALTH AND DISEASE}

A breakthrough discovery in 2004 showed that structural genomic variants (CNVs) are, as a class, common in the general population. ${ }^{31,32}$ These include both losses (deletions) and gains (duplications) with diverse potential mechanisms of action (Figure 2). ${ }^{33}$ CNVs are not inherently pathological. Within any individual's genome, there are typically both myriad common (i.e., $>1-5 \%$ frequency in the general population), and one or more rare, CNVs. The mutation rate of structural rearrangements like CNVs is much higher than that of single base pair changes, and CNVs may disrupt none, one, or several genomic elements (Figure 2). ${ }^{31-33}$ Some 'recurrent' CNVs are individually rare but arise with similar breakpoints in unrelated families because of an underlying genomic architecture that predisposes to CNV. CNVs that are common may act as neutral variants or sometimes as 
a
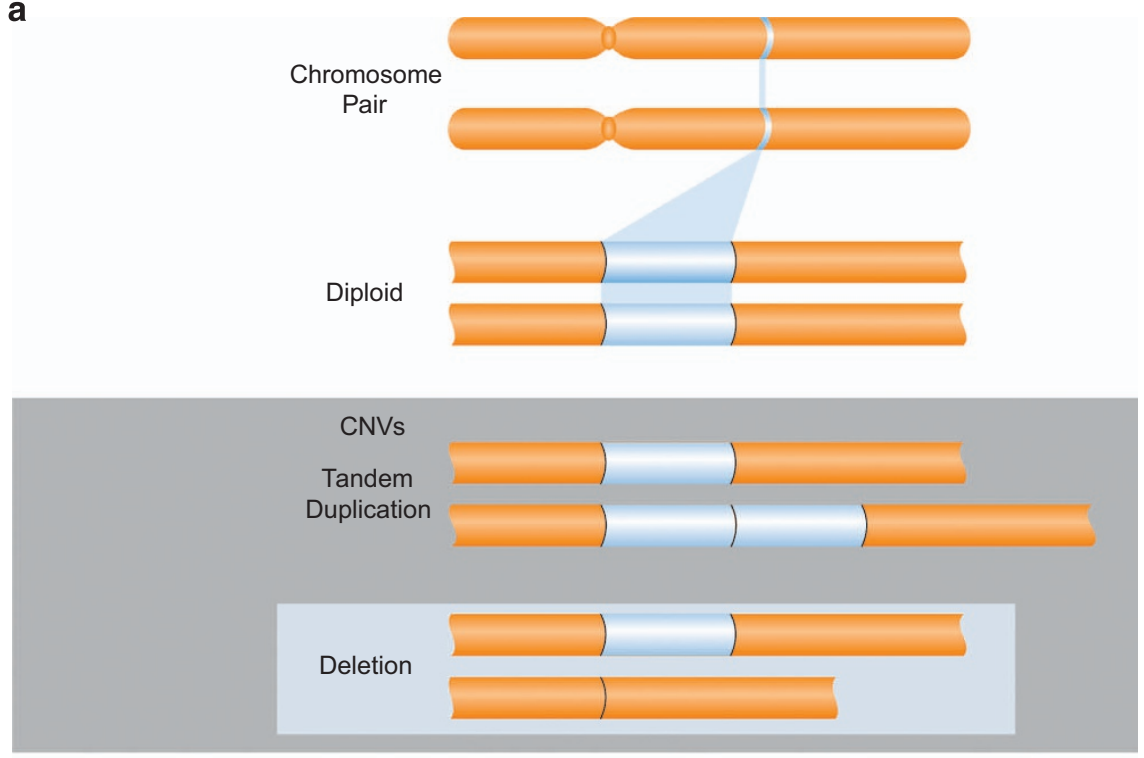

b

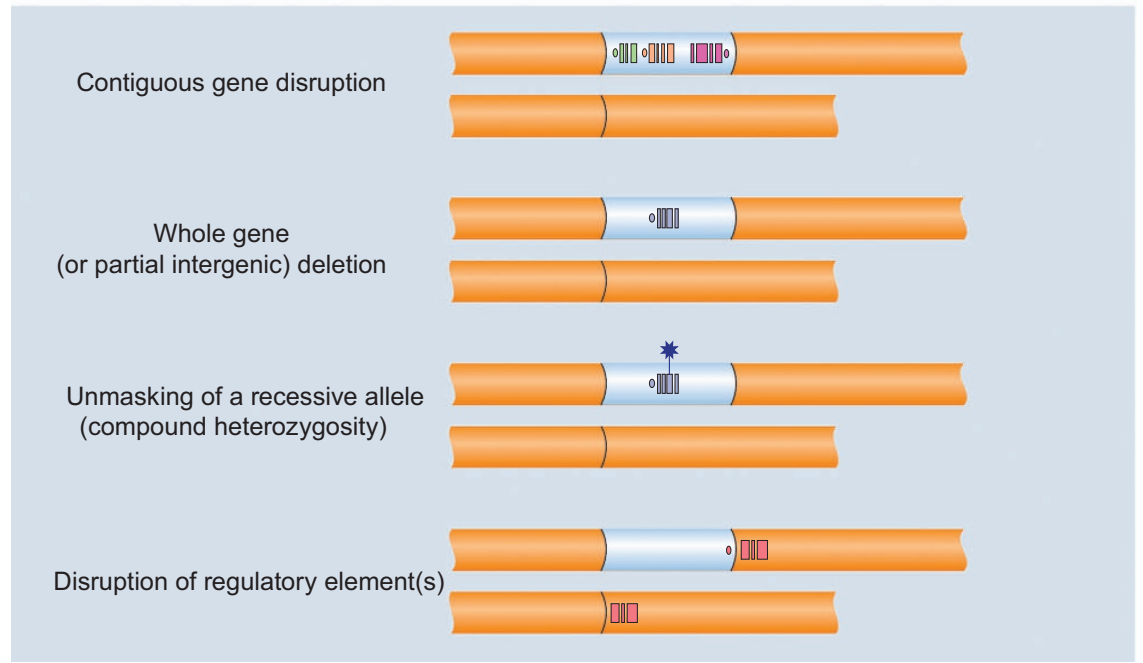

Figure 2. Examples of CNV and associated disease mechanisms. (a) Normal diploid status and two examples of CNV (a simple deletion and a tandem duplication). Not pictured are the diverse other forms of CNV, including non-contiguous insertions, higher-order copy number changes (multi-allelic CNV), and more complex rearrangements. CNVs may involve no, one or multiple genomic elements. (b) Selected mechanisms underlying disease effects of copy number losses (deletions). A gene is indicated by a contiguous monochromatic set of rectangles, and a regulatory element (e.g., promoter) by an oval. The definition of 'gene' extends beyond protein-coding genes to potentially include noncoding elements like microRNAs and long noncoding RNAs. Of note, duplications can effect change through increased copy number of a dosage sensitive gene (not pictured) or via the mechanisms depicted for deletions (e.g., via disruption at a breakpoint or partial intragenic duplication). Inspired by Figure 1 in ref. 92 and Figure 2 in ref. 33.

modifiers of disease susceptibility. Rare CNVs are far more likely than common CNVs to be associated with disease, especially developmental disorders like $\mathrm{CHD}^{25,33-36}$ As for virtually all genetic variants, CNVs, including rare pathogenic CNVs, are usually associated with some degree of variable expression and/or incomplete penetrance. Increasing knowledge about CNV has revolutionized thinking about differences between individual human genomes and the genetic architecture of common complex (multifactorial) disorders like $\mathrm{CHD}{ }^{25}$

\section{GENOME-WIDE CNV IN CHD}

Over the past decade strong evidence has accumulated that genome-wide rare CNV represents a considerable source of the genetic variation that contributes to CHD susceptibility. ${ }^{37-56}$ These findings include both known associations such as chromosome 22q11.2 deletions, and new discoveries across the genome. Studying CNV in CHD is contributing to our understanding of cardiac morphogenesis and related disorders, ${ }^{25}$ and to new knowledge that is relevant for clinical practice.

Increased overall burden of rare CNVs

A consistent finding across multiple genome-wide studies is that there is an excess burden of rare CNVs in CHD compared with control populations (Table 1). ${ }^{38-40,42-55,57,58}$ Differences in the definition of rarity, size cut-offs, overlap of genes and/or coding sequence (exons), array platforms and the CNV calling algorithm(s) used, other technologies, exclusions (e.g., karyotypic anomalies, 22q11.2 deletions) and CHD sample ascertainment strategies, will all affect the proportions of individuals reported to have rare CNVs. The majority of patients studied were putatively non- 
Table 1. Case-control studies of genome-wide rare CNV burden in $\mathrm{CHD}^{\mathrm{a}}$

Study Cases CHD type Controls Significant case-control CNV burden findings

22q11.2

(n) (n)

\begin{tabular}{|c|c|c|c|c|c|}
\hline Zhao et al. ${ }^{43}$ & 100 & Various $^{\mathrm{b}}$ & 65 & Increased proportion of subjects with rare CNVs $>100 \mathrm{~kb}$ in size (39.0 vs $21.5 \%$ ) & $Y_{e s}^{c}$ \\
\hline Costain et al. ${ }^{53}$ & $101^{\mathrm{d}}$ & TGA & $415^{\mathrm{e}}$ & Increased proportion of subjects with rare CNVs $>500 \mathrm{~kb}$ in size (10.1 vs $4.6 \%$ ) & Yes \\
\hline Carey et al. $^{58}$ & 223 & $\begin{array}{l}\text { Single } \\
\text { ventricle }\end{array}$ & 270 & Increased proportion of subjects with rare genic CNVs $>300 \mathrm{~kb}$ in size (13.9 vs $4.4 \%$ ) & Yes \\
\hline Fakhro et al. ${ }^{48}$ & 262 & HTX & 991 & Increased proportion of subjects with rare genic CNVs (14.5 vs $7.4 \%)$ & Yes \\
\hline Kim et $a l^{54}$ & 422 & Various ${ }^{\mathrm{b}}$ & 500 & Increased proportion of subjects with rare genic CNVs $>300 \mathrm{~kb}$ in size (12.1 vs $5.0 \%)$ & Yes \\
\hline Silversides et al. $^{38}$ & $433^{d}$ & TOF & $416^{\mathrm{e}}$ & Increased proportion of subjects with rare CNVs $>500 \mathrm{~kb}$ in size $(9.1 \text { vs } 5.1 \%)^{\mathrm{f}}$ & Yes \\
\hline Soemedi et al. ${ }^{39}$ & 2,256 & Various $^{\mathrm{b}}$ & 841 & Increased proportion of subjects with rare genic loss CNVs ( 7.8 vs $4.4 \%$ ) & Yes \\
\hline
\end{tabular}

Abbreviations: ASD, atrial septal defect; CHD, congenital heart disease; CNVs, copy number variations; HLHS, hypoplastic left heart syndrome; HTX, heterotaxy; PDA, patent ductus arteriosus; TGA, transposition of the great arteries; TOF, tetralogy of Fallot; VSD, ventricular septal defect.

a Minimum $n=100$ case subjects. Glessner et al. ${ }^{57}$ reported an increased burden of de novo CNVs in CHD cases relative to controls, but multiple numerical inconsistencies in their report resulted in its exclusion from this table.

bZhao et al. ${ }^{43}$ : ASD $(n=58)$, VSD $(n=22)$, PDA $(n=15)$, TOF $(n=2)$, Ebstein anomaly $(n=2)$, and tricuspid incompetence $(n=1)$; Kim et al. ${ }^{54}$ : HLHS $(n=130)$, TOF $(n=64)$, TGA $(n=34), \operatorname{VSD}(n=40), \mathrm{VSD} / \mathrm{CoA}(n=19)$, single ventricle $(n=30)$ and other $(n=105)$; Soemedi et al. ${ }^{39}$ : the four largest of the 29 categories were TOF $(n=808)$, ASD $(n=293)$, TGA $(n=165)$ and VSD $(n=163)$.

cIncluded one atypical 22q11.2 deletion overlapping CRKL.

dOnly subjects of European ancestry were considered in burden analyses.

${ }^{\mathrm{e}} \mathrm{All}$ rare CNVs in cases and controls were adjudicated for rarity by comparing to those in additional population-based controls: $n=2357,{ }^{38} n=10113 .{ }^{53}$

${ }^{\mathrm{f} A l s o}$ within-TOF finding of more exonic losses in the syndromic subgroup.

Table 2. Studies of genome-wide de novo CNV rate in CHD (22q11.2 deletions excluded) ${ }^{a}$

\begin{tabular}{|c|c|c|c|c|c|c|}
\hline \multirow{2}{*}{$\begin{array}{l}\text { Study } \\
\text { Hitz et al. }{ }^{40}\end{array}$} & \multirow{2}{*}{$\begin{array}{l}\text { Recruitment site(s) } \\
\text { Canada (QC) }\end{array}$} & \multirow{2}{*}{$\begin{array}{c}\text { Case trios (n) } \\
53\end{array}$} & \multirow{2}{*}{$\begin{array}{l}\text { CHD type } \\
\text { Left-sided }\end{array}$} & \multirow{2}{*}{$\begin{array}{l}\text { Array type(s) } \\
\text { Affymetrix Human Genome-Wide SNP Array } 6.0 \\
\text { (Santa Clara, CA, USA) }\end{array}$} & \multicolumn{2}{|c|}{$\begin{array}{l}\text { De novo rate } \\
\text { per subject }\end{array}$} \\
\hline & & & & & $6 / 53$ & $11.3 \%$ \\
\hline Xie et $a .^{42}$ & South Central China & 82 & PA & $\begin{array}{l}\text { Illumina 660W-Quad \& Omni1-Quad BeadChips } \\
\text { (San Diego, CA, USA) }\end{array}$ & $12 / 78$ & $15.4 \%$ \\
\hline Greenway et al. ${ }^{45}$ & USA (Boston), Brazil & 114 & TOF & Affymetrix Human Genome-Wide SNP Array 6.0 & 9/112 & $8.0 \%$ \\
\hline Warburton et al. ${ }^{47}$ & USA (NY) & 223 & CNT, HLHS & NimbleGen CGH HD2 (Madison, WI, USA) & $20 / 213$ & $9.4 \%$ \\
\hline \multirow{2}{*}{ Sanchez-Castro et al. ${ }^{55}$} & & & TOF & & $5 / 81$ & $6.2 \%$ \\
\hline & & & TGA & & $0 / 159$ & $0.0 \%$ \\
\hline Glessner et al. ${ }^{57}$ & USA (various) & 538 & Various $^{c}$ & Illumina Omni-1.0 and $2.5 \mathrm{M}^{\mathrm{d}}$ & $47 / 534$ & $8.8 \%$ \\
\hline $\begin{array}{l}\text { heart syndrome; HTX, he } \\
\text { a Minimum } n=50 \text { trios. } \\
\text { bProportion of unrelated } \\
\text { cLeft-ventricular outflow } \\
\text { d Also employed whole-e) }\end{array}$ & $\begin{array}{l}\text { rotaxy; PA, pulmonary atresia; } \mathrm{TC} \\
\text { ase subjects with at least one de } \\
\text { sions }>\mathrm{CNT} \gg \text { heterotaxy }>\text { other } \\
\text { me sequencing for characterisat }\end{array}$ & $\begin{array}{l}\text { hovo CNV, af } \\
\text { (exact numb } \\
\text { n of CNV. }\end{array}$ & $\begin{array}{l}\text { excluding in } \\
\text { cannot be }\end{array}$ & $\begin{array}{l}\text { ndividuals with } 22 q 11.2 \text { deletions. } \\
\text { determined from data provided). }\end{array}$ & & ct. \\
\hline
\end{tabular}

syndromic. Most studies excluded 22q11.2 deletions and other diagnosable syndromes (Table 1). This may obscure the fact that a relatively large proportion of individuals with CHD have $22 q 11.2$ deletions. ${ }^{29,59}$ TOF is the best-studied cardiac lesion but all CHD adequately studied to date shows an excess burden of rare CNVs. Both gain and loss CNVs are involved, with certain loci recurring (see below), in addition to the 22q11.2 deletion. One attempt at a meta-analysis identified dozens of putatively associated loci. ${ }^{52}$ Other CNVs appear so rarely that recurrence has not yet been observed. Most studies have reported only CNVs involving autosomes; sex chromosome copy number findings may also be relevant to $\mathrm{CHD}$. $^{60}$

Increased burden of de novo CNVs

Restricting to rare CNVs that have arisen as de novo mutations shows similar excess burden findings in CHD, even after excluding 22q11.2 deletions (Table 2). Few studies employed direct control populations; however, the general population rate of de novo CNVs is well studied in other cohorts and typically quoted as $<2 \%{ }^{47,57}$ One study involving patients with TGA reported a lower de novo mutation rate than for other cardiac lesions. ${ }^{55}$ This would be consistent with offspring recurrence data for TGA that suggest fewer dominant-acting mutations for this $\mathrm{CHD}^{53}$ However, the numbers of patients studied to date is too small to draw firm conclusions (Table 2). The elevated de novo CNV mutation rate in other severe CHD may in part explain how it is maintained in the population, despite a strong negative selective pressure and before the advent of modern paediatric surgery. Although rare de novo mutations are of interest and often considered to be more highly penetrant, it is important to note that most CNVs, even rare CNVs known to be pathogenic, are inherited. Moreover, recurrent CNVs that arise de novo in some patients are inherited from 
Box 1 Genetic studies of 22q11.2 deletion syndrome (22q11.2DS) as a guide for understanding variable expression

The 22q11.2 deletion alone cannot account for the variable expression of the congenital cardiac phenotype in 22q11.2DS. ${ }^{29}$ Efforts are underway to dissect the layered genetic architecture that gives rise to this variability. Progress to date offers a blueprint for studying pathways from CNV genotype to CHD phenotype more generally.

\section{Within the 22q11.2 region:}

- Length of the deletion and specific breakpoints

- There is little to no discernible association of severity of the phenotype, including the CHD, with the length of the deletion. $^{113}$

- Animal models of the full deletion and of haploinsufficiency of specific deleted genes

- Haploinsufficiency of multiple genes within the region appears to be important, including $T B X 1^{30} C R K L^{114}$ and DGCR8. ${ }^{115}$

- Variation on the intact allele

- Sequence variation in TBX1 on the intact $22 q 11.2$ allele does not have a major role in cardiac expression. ${ }^{116}$

Outside the 22q11.2 region (genome-wide):

- Family studies

- Family history data support a heritable susceptibility to CHD in families of probands with de novo 22q11.2 deletions and CHD. ${ }^{117,118}$

- Common genome-wide variation

- A common duplication of SLC2A3 was significantly enriched in individuals with 22q11.2 deletions and CHD, implicating this CNV as a possible genetic modifier in a small proportion of subjects. ${ }^{113}$

- Additional rare CNV

- Preliminary data suggest that rare CNVs outside the 22q11.2 region may overlap genes that modify risk for CHD in a small proportion of subjects. ${ }^{119}$

- Additional rare sequence mutations

- Whole-exome sequencing implicated rare mutations in histone modification genes as possibly influencing risk for CHD in the presence of the $22 q 11.2$ deletion. ${ }^{120}$

parents without CHD in others (see below), consistent with variable expression and in some cases reduced penetrance.

\section{$22 q 11.2$ deletions and duplications}

The role of 22q11.2 deletions in CHD was well delineated before the advent of chromosomal microarray analysis (CMA). ${ }^{29}$ Cumulative prevalence estimates of this recurrent 22 q11.2 deletion include $\sim 50 \%$ in interrupted aortic arch type $B, \sim 33 \%$ in truncus arteriosus, $\sim 15 \%$ in TOF and $5-10 \%$ in VSD. ${ }^{29,59}$ Appreciation for other genome-wide rare recurrent and non-recurrent CNVs in CHD more generally has served to reinforce the importance of $22 \mathrm{q} 11.2$ deletions as the archetypal model for continued genetic discovery and clinical translation of findings. From a molecular genetic perspective, human and animal studies are shedding light on the specific determinants of cardiac expression (Box 1). From a clinical

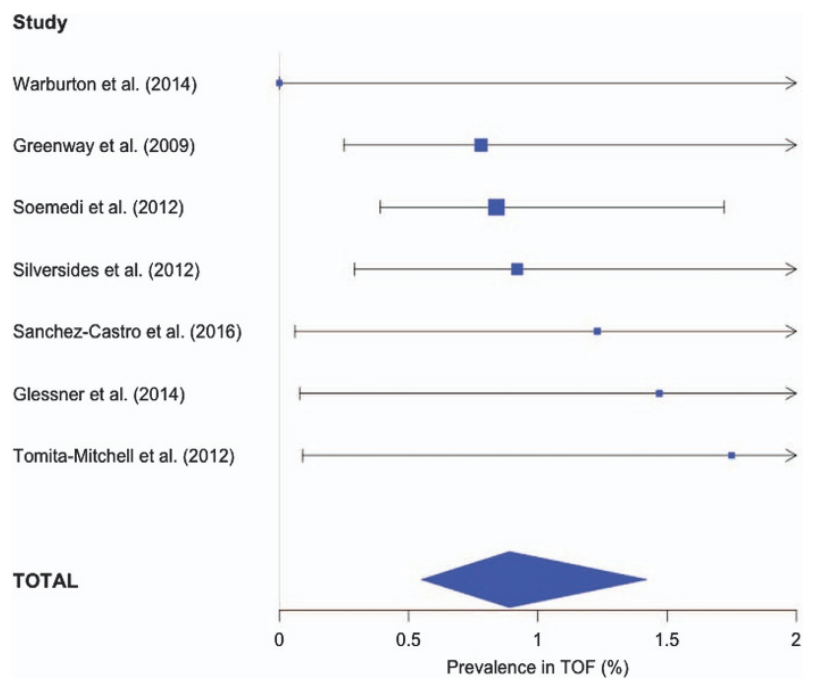

Figure 3. Prevalence of $1 \mathrm{q} 21.1$ duplications in cohorts with TOF. Box size is proportional to study size: $n=33,{ }^{47} n=510,{ }^{45} n=948,{ }^{68}$ $n=433,{ }^{38} n=81,{ }^{55} n=68^{57}$ and $n=57.0^{50}$ The blue diamond represents the combined prevalence of 1q21.1 duplications in a total of 2130 subjects with TOF, and the diamond width corresponds to $95 \%$ confidence interval bounds. In contrast, 1q21.1 duplications are rare in control populations (see text). ${ }^{67}$

perspective, diagnosis is helpful and changes management, including genetic counselling. ${ }^{29,61,62}$

General features of $22 q 11.2$ deletions are proving to be generalisable to other CNVs associated with CHD. Although enriched for conotruncal and other anomalies, all types of CHD have been associated with 22q11.2 deletions. Severity ranges from non-viable (fetal and early pregnancy loss) to subclinical, e.g., spontaneously closing VSDs. Many patients do not have a CHD phenotype. If ascertainment is not through congenital cardiac clinics, the prevalence of readily detectable CHD may be as low as $\sim 25-40 \%{ }^{63}$ Although multi-system expression is the norm over the lifetime, readily detectable congenital anomalies may not be present. $^{29}$ The CHD may appear 'isolated', especially prenatally or at birth, as neurodevelopmental and neuropsychiatric features may not become apparent for years or decades. ${ }^{29,61,62}$ Dysmorphic features are usually subtle, and absence of 'typical' facial features does not affect the likelihood of the diagnosis being present or the severity of the presentation. ${ }^{29}$ Mortality is significantly increased, ${ }^{29}$ and surgical and perioperative complications in those with 22q11.2 deletions and conotruncal anomalies can be greater than other patients with the same CHD. ${ }^{64}$ The deletion may be inherited or de novo. If inherited, the parent with the deletion will often not have CHD or, if present, the same CHD or other features as the affected proband. ${ }^{29}$ There is a high mutation rate in the population due to the local genomic architecture: flanking segmental duplications and resulting increased risk of non-allelic homologous recombination during meiosis. The reciprocal duplication can result in similar phenotypes, including CHD. ${ }^{29,65}$

\section{1q21.1 deletions and duplications}

One of the first loci to be identified from genome-wide studies of CNV in CHD was the recurrent 1q21.1 CNV. Sometimes occurring as a deletion, ${ }^{66}$ but more commonly as a duplication, this is a highly replicated finding, especially in TOF where the prevalence appears to be just less than one in every 100 patients (Figure 3). ${ }^{38,39,45,47,50,55,57,67,68}$ As for all CNVs associated with CHD, the expression is variable, even within families, for both cardiac anomalies and other phenotypes, and is not necessarily 
correlated with the length of the CNV. ${ }^{38,40,47,52,67,69} 1 \mathrm{q} 21.1$ duplications are rare in control populations, e.g., in five out of 18,828 controls $(0.027 \%)$ from three studies, where some controls were not screened for disease (reviewed in ref 67). Although it is uncertain if any single gene at this locus can be designated as truly causal, the most evidence points to the GJA5 gene that encodes connexin $40,{ }^{38,68}$ a cardiac gap junction protein expressed in the right ventricular outflow tract. ${ }^{45}$ Point mutations in GJA5 can be associated with atrial fibrillation. ${ }^{70}$

\section{8p23.1 deletions overlapping GATA4}

Before the first wave of genome-wide CNV studies, the 8p deletion syndrome and sequence mutations in GATA4 were both reported to be associated with CHD. ${ }^{14,71,72}$ Haploinsufficiency of GATA4 is now one of the most frequent rare structural anomalies identified in genome-wide CNV studies of CHD. ${ }^{34,39,41,47,48,50,52,58}$ Unlike the typical chromosomal rearrangements at $1 \mathrm{q} 21.1,15 q 11.2$ and 22q11.2 mediated by non-allelic homologous recombination, these $8 p$ deletions are non-recurrent and of varying size. Haploinsufficiency of adjacent genomic elements like SOX7 may shape the cardiac expression. ${ }^{41,73}$ Duplications at the 8 p23.1 locus have also been reported in CHD. ${ }^{48,50,58,60}$ The spectrum of cardiac lesions favours septal defects, including atrioventricular canal defects, ${ }^{52}$ but as for other loci there is significant cardiac phenotypic heterogeneity. GATA4 encodes an essential cardiac transcription factor that works in concert with other key regulators of cardiac morphogenesis like NKX2-5 and TBX5. ${ }^{14,71}$

\section{$15 q 11.2$ deletions}

The putative association of $15 q 11.2$ deletions with CHD illustrates the complexity imbued by incomplete penetrance, non-cardiac phenotypic expression, and ascertainment. Proximal deletions in this complex region (breakpoints BP1-BP2) on chromosome 15 are risk factors of modest effect for neurodevelopmental and neuropsychiatric disorders. ${ }^{34,74-76}$ A majority are inherited, ${ }^{74}$ typically from a parent with milder or no overt phenotypic consequences. With a nonspecific phenotype and prevalence in control populations of up to 1 in $400,{ }^{75} 15 q 11.2$ deletions are typically categorized as variants of uncertain significance (VUS).

Two early studies reported that individuals with this $15 q 11.2$ deletion were moderately enriched in cohorts with diverse CHD, relative to control populations. ${ }^{34,39}$ However, one study had an increased burden of extra-cardiac phenotypes in cases, ${ }^{34}$ and the other had a low prevalence in the controls used (one in 1,538). ${ }^{39}$ Since then, 15 q11.2 deletions have been identified infrequently in genome-wide CNV studies of $\mathrm{CHD}^{57}$ Case series of individuals with $15 q 11.2$ deletions ascertained through clinical testing for developmental phenotypes or multiple congenital anomalies report variable rates of $\mathrm{CHD},{ }^{74-76}$ influenced in part by screening with echocardiography. ${ }^{74}$ A notable proportion have had left-sided cardiac lesions, ${ }^{39,57,74,75}$ but the observed spectrum of cardiac lesions is broad. Specific susceptibility element(s) within this region remain to be determined. The typical deletion encompasses four highly conserved, non-imprinted genes (CYFIP1, NIPA1, NIPA2 and TUBGCP5) with as yet no independent link to cardiac morphogenesis. Additional rare variants are common in these individuals and may act independently or in concert with the $15 q 11.2$ deletion to increase risk for $\mathrm{CHD}^{75-77}$ although in one case series there was similar prevalence of CHD in a smaller subgroup without secondary CNVs. ${ }^{76}$

\section{Pathway analyses and gene families}

Interpreting genome-wide CNVs is challenging, as individual variants are rare and often involve multiple genomic elements. Also, non-knockout mutation dosage changes in most genes are uncharacterized in both humans and model organisms. Several studies have applied pathway and gene enrichment analyses of varying degrees of sophistication to rare CNV datasets to identify gene sets important to cardiac development. Although most were limited in scope, there are significant results for not only functional gene sets that would be expected (vascular development/cardiac structure ${ }^{47}$ but also for more novel gene sets: WNT signalling, ${ }^{39}$ 'gene neighbours' (with shared genetic or physical interactions) of GATA4/TBX5/NKX2-5, ${ }^{38,57}$ angiogenesis, ${ }^{40}$ semaphorin-plexin pathways, ${ }^{38}$ ciliary proteome, ${ }^{38,48}$ TGF-beta signalling ${ }^{48}$ and neuron projection. ${ }^{38}$ The latter is notable because of the high rate of neurodevelopmental problems in patients with $\mathrm{CHD}$, and similar findings from a large whole-exome sequencing study. ${ }^{78}$ There is also overlap with arrhythmogenic genes initially implicated in electrical/functional as opposed to structural heart abnormalities (e.g., GJA5 and CACNA1C). ${ }^{38,53,68,77}$ CNV overlap of non-protein-coding genes like microRNAs is less well studied but may also implicate cardiac pathways. ${ }^{79,80}$

\section{CLINICAL GENETIC TESTING WITH CMA}

Diagnosis of microdeletion and microduplication syndromes previously necessitated a high index of suspicion for classic clinical features that may or may not be present. CMA (also known as array comparative genomic hybridisation) is a clinical genetic test that allows for the identification of not only the individually rare CNVs underlying these established syndromes but also other emerging 'genomic disorders' across the genome. Initial consensus testing indications for CMA were multiple congenital anomalies and/or developmental delay/intellectual disability/ autism spectrum disorder. ${ }^{35}$ Various outcomes are possible with CMA (Box 2).

The clinical yield of CMA in CHD has been best described in the prenatal setting. Jansen et al. ${ }^{81}$ identified 13 publications that included 1,131 cases in their meta-analysis of array comparative genomic hybridisation studies in prenatally diagnosed CHD. The incremental yield (i.e., excluding aneuploidy and 22q11.2 deletions) of clinically relevant CNVs was 7.0\%; $3.4 \%$ in 'isolated' CHD and $9.3 \%$ in syndromic CHD. ${ }^{81}$ When including $22 q 11.2$ deletions, the overall yield was $12 \%$ (about one in eight). ${ }^{81}$ An additional benefit of CMA was in the detection of atypical $22 q 11.2$ deletions that are not detectable using standard probes in targeted fluorescence in situ hybridisation testing. VUS were identified in an additional $3.4 \%$ of CHD fetuses. ${ }^{81}$ Thus for pre-test counselling, one might predict a $14 \%$ chance of uncovering an anomaly on CMA that is deemed clinically reportable: $4 \% 22 q 11.2$ deletion, $7 \%$ other pathogenic CNV and 3\% VUS.81 One caveat is that pathogenic CNV included both those variants implicated in $\mathrm{CHD}$, as well as 'incidental' findings relevant to neurodevelopment. The largest component study ${ }^{82}$ drove most of the significant findings in this meta-analysis. As more knowledge accumulates, original results of such genetic testing need to be re-annotated with respect to pathogenicity. Postnatal studies support a similarly high and increasing yield of pathogenic CNVs in both isolated and syndromic CHD cohorts, ${ }^{83,84}$ as well as a high rate of VUS. ${ }^{85}$ As expected, the yield is consistently higher in individuals with syndromic CHD. ${ }^{51,83}$

Indications for genome-wide testing are likely to expand. In practice, CMA is now often offered for apparently isolated forms of serious CHD. ${ }^{84,85}$ Some have advocated for the universal postnatal use of CMA in isolated CHD. ${ }^{86}$ In consanguineous families, single-nucleotide polymorphism arrays offer the added benefit of determining regions of loss of heterozygosity, which may then direct targeted sequencing of recessive genes implicated in cardiovascular development. The cost of testing and the current high rate of VUS are important disadvantages. Another major caveat is that most single gene disorders are not detectable with CMA. Patients, families and clinicians may mistakenly conclude on the basis of a normal CMA result that an identifiable genetic 
Box 2 Outcomes of clinical genetic testing with CMA

There are four general outcomes of CMA: (1) no abnormality (normal array); (2) pathogenic variant; (3) VUS; and, rarely, (4) secondary or incidental finding. Categories 2-4 may co-occur. Many laboratories follow the American College of Medical Genetics and Genomics (ACMG) guidelines for interpretation and reporting of postnatal constitutional CNVs. ${ }^{121}$

(1) Normal array: In the case of a normal array, the cause of the individual's CHD remains unexplained. Counselling about a negative result includes the fact that this is the likeliest outcome. This normal result does not preclude the likelihood that other genetic testing may be clinically available now or in the future that could help to resolve the aetiology of the CHD. CMA will not detect sequence mutations, or chromosomal imbalances below a certain resolution.

Example: A normal array in a patient with truncus arteriosus and a loss-of-function mutation in NKX2-5.

(2) Pathogenic variant: Discovery of a pathogenic CNV that is a known risk factor for CHD will prompt a recommendation for a referral to medical genetics and/or a sub specialty clinic for further specific assessment and counselling. Parental testing where possible is usually recommended. In some cases, parental studies will uncover the same CNV or perhaps a balanced chromosome rearrangement that confers a significant recurrence risk for having other children with a pathogenic CNV.

Example: A typical 1q21.1 duplication in a patient with TOF. ${ }^{67}$

(3) VUS: VUS are those CNVs that are not yet reliably characterised as either benign or pathogenic. Subcategories exist: 'VUS; likely pathogenic', 'VUS; likely benign' and 'VUS (no sub-classification)'. ${ }^{121}$ Genetic assessment and counselling may be recommended about what is known now, and/or in the future should new information arise. VUS, especially those deemed 'likely pathogenic', are often a source of future pathogenic variants.

Example: A duplication overlapping CACNA1C and a typical $15 q 11.2$ deletion in a patient with TGA. ${ }^{77}$

(4) Secondary finding: There is the possibility of finding a variant associated with a genetic disorder not believed to be related to the primary reason for referral. In these rare instances, the genetic disorder may be associated with an adult onset condition.

Example: Haploinsufficiency of the familial breast and ovarian cancer gene BRCA2 in a patient with TOF. ${ }^{96}$

contribution to the CHD has been ruled out (Box 2). The application of CMA, while helpful for providing a molecular diagnosis for a significant minority of patients with $\mathrm{CHD}$, is not a replacement for a comprehensive medical genetic evaluation.

\section{RECURRENCE RISK ESTIMATION AND GENETIC COUNSELLING}

Genetic counselling in CHD has traditionally relied upon empiric recurrence risk values, ${ }^{87,88}$ but rare CNVs can have a significant impact in specific cases. As in other domains, classic microdeletion syndromes like 22q11.2DS are the archetypal example of clinical applicability. Other genetically diagnosable subtypes of CHD promise similar possibilities with respect to changing standard recurrence risk prediction and genetic counselling. ${ }^{67,87,88}$

Incorporating rare CNVs into risk prediction and counselling requires a nuanced approach. Careful phenotyping and genotyping of the parents is essential. ${ }^{87} \mathrm{~A}$ de novo CNV may be mistaken for the sole, causal variant in an individual with CHD. In one example, a subject with left-sided $\mathrm{CHD}$ and a de novo partial 1q21.1 duplication also inherited two rare CNVs overlapping candidate genes for CHD from a less severely affected father. ${ }^{40}$ De novo rare CNVs can seemingly have no, or subclinical deleterious effects. Parental germline mosaicism is also a consideration. In one recent study of rare CNV in CHD, the parents of two out of 20 affected probands with apparently de novo CNVs were ultimately found to have low level mosaicism in serum. ${ }^{47}$

Most rare CNVs are inherited. Some, such as 22q11.2 deletions, are recurrent and/or have been observed in unrelated individuals, while others are ultra-rare or unique to a particular family (private). Inheritance of a CNV from a purportedly unaffected parent can be falsely reassuring. As discussed above, variable expression of cardiac phenotypes is the norm. The effects of high but incompletely penetrant mutations on reproductive fitness in the parental generation are an important source of bias. ${ }^{89,90} \mathrm{~A}$ parent may also have a subclinical cardiac phenotype (e.g., bicuspid aortic valve demonstrates familial recurrence with hypoplastic left heart syndrome ${ }^{91}$ ) or a non-cardiac phenotype that may not be readily apparent. Alternatively, inheritance of any variant from an affected parent has an a priori likelihood of at least 50\%. Cosegregation of a variant with CHD within a nuclear family (i.e., from affected parent to affected child) is thus suggestive but not specific. Two large-scale studies have considered multiplex nuclear families, ${ }^{40,47}$ but none to date have adjudicated CNVs within the extended family context. Counselling always needs to emphasise the potential for considerable variability in the type and severity of CHD, and in any extra-cardiac manifestations.

Prognostication in paediatric probands and concerns about sibling recurrence were previously the primary impetus for clinical genetic testing and counselling. With improved childhood survival, there is growing interest in issues germane to adults with $\mathrm{CHD}^{10-12}$ including offspring recurrence. ${ }^{19,88}$ Irrespective of informative molecular genetic findings, surveillance during pregnancy, including fetal echocardiography, is the standard of care for offspring of both women and men with CHD. In the setting of a putative susceptibility CNV in either parent, there may be opportunities for pre-implantation genetic diagnosis or prenatal genetic diagnosis (with chorionic villus sampling or amniocentesis). The above caveats about interpreting inherited variants are relevant in this situation. Whether genetic subtyping with respect to rare CNV could also inform general obstetrical management in women with $\mathrm{CHD}$ is a question that requires further study.

\section{CLINICAL PROGNOSTICATION AND PREDICTION OF EXTRA-CARDIAC FEATURES}

Rare CNVs are associated with a range of developmental disorders and major extra-cardiac phenotypes. ${ }^{33,34,92}$ Available data suggest that 'syndromal' patients with TOF, including those with recognisable genetic syndromes, have worse surgical outcomes and worse 10-year actuarial survival. ${ }^{93,94}$ Gaynor et al. ${ }^{95}$ showed that neurodevelopmental outcomes were also significantly worse in infants with CHD who had confirmed or suspected genetic syndromes. Notably, patient-specific factors were more important predictors of worse neurodevelopmental outcomes than intraoperative factors. ${ }^{95}$ There is a clinical impetus to better understand and predict phenotypic expression of rare CNVs across the lifespan, particularly as regards surgical and neurodevelopmental outcomes. In general, the larger and rarer the rearrangement the more easily observable the phenotypic effects. $^{33}$ Given comparably sized rearrangements at the same locus, deletions usually have more severe phenotypic effects than duplications. $^{33}$ Last, extra-cardiac features can appear later in life. ${ }^{61,77,92,96}$ For this reason, the 'syndromic' label is difficult to 
apply reliably and may be misleading, particularly in children and in the prenatal setting.

Two studies have explored the potential consequences of large rare CNVs on early outcomes, after excluding aneuploidies and 22q11.2 deletions. Carey and colleagues studied 223 patients with single-ventricle CHD and data on neurocognitive and growth outcomes at 14 months. ${ }^{58}$ They identified putatively pathogenic rare genic CNVs $>300 \mathrm{~kb}$ in size in 14\% (25 duplications and six deletions), including CNVs associated with genomic disorders in 13 patients. Comparing those with a deletion or duplication (CNV+) to those without such a CNV showed some subtle effects on growth associated with duplications, and worse neurocognitive outcomes on one of two measures in the small subgroup with deletions. Of note, worse neurodevelopmental outcomes are associated with large deletions in other populations without CHD. ${ }^{97,98}$ As anticipated, worse neurocognitive outcomes were associated with the genomic disorders associated with recurrent CNVs. ${ }^{58}$ Only three out of the 14 subjects examined in the CNV+ group had dysmorphic features or significant extra-cardiac phenotypes. ${ }^{58}$ Recently, Kim et al. ${ }^{54}$ reported that the presence of a rare genic CNV $>300 \mathrm{~kb}$ in size was also independently associated with decreased transplant-free survival in a separate cohort of 422 children with non-syndromic CHD.

With increasing survival into adulthood, genetic factors that may inform longevity and reproduction are clinically, as well as scientifically, relevant. Late cardiac complications result in significant morbidity and mortality; however, not all patients develop adverse late outcomes (cardiac or extra-cardiac). ${ }^{10-12,99}$ Adults with 22q11.2DS, with and without CHD, have been studied. ${ }^{89,100}$ One study of reproductive fitness in adults with TOF and without 22q11.2DS showed that syndromal subjects were more likely to be childless, but failed to identify any association with the rare CNV profile. ${ }^{19} \mathrm{~A}$ smaller adult cohort with TGA, ascertained and studied in a similar manner, showed comparable results. $^{53} \mathrm{~A}$ majority of deaths in adults with $\mathrm{CHD}$ are a consequence of cardiovascular complications, including heart failure, arrhythmias and sudden death. ${ }^{10-12,99}$ Whether rare CNV burden is also associated with later clinical outcomes is unknown.

\section{INSIGHTS INTO HUMAN CARDIAC DEVELOPMENT, AND IMPLICATIONS FOR DISCOVERY-BASED SCIENCE}

Several genetic mechanisms and many genes are involved in increasing susceptibility to CHD. Microarray technology for genome-wide CNV detection has greatly accelerated the pace of discovery of new candidate genes and pathways. Ongoing research to delineate copy number variable regions in the genome and measure their frequencies in the general population, at ever-higher resolutions, is key to being able to interpret results from disease populations. Efforts to catalogue CNV loci associated with CHD are valuable (see, e.g., the meta-analysis by Thorsson et $a .^{52}$ ), but any such list requires regular updating and revision.

CNVs can narrow critical regions for CHD, including those previously implicated by large karyotypically-visible anomalies. Hundreds to thousands of protein-coding genes are expressed in the human heart or great vessels at some point in early development. The complex interplay of multiple proteins and regulatory elements may lead to relative weak spots susceptible to disruption by mutations, yet this susceptibility is balanced by the innate redundancy of these systems. Disruption of normal cardiac morphogenesis requires overcoming the robustness of genetic networks that have been fine-tuned through evolution. By virtue of their size and mutational frequency, CNVs can simultaneously disrupt multiple genes and/or regulatory elements within an individual (Figure 2), and this could be why CNV is so important to CHD causation. In addition to the examples provided above, studies involving the 1 p36 deletion syndrome nicely illustrate this concept, as well as the associated challenges in ascribing causality to specific genes. ${ }^{101-105}$ Only a minority of rare CNVs however will result in complex multi-system developmental phenotypes and/or in utero or early neonatal mortality.

The considerable variability in the type and severity of CHD associated with individual recurrent CNVs provides further support for, and new insights into, the genetically and functionally interdependent pathways that govern cardiogenesis. There appears to be significant shared underlying genetic susceptibility to lesions considered anatomically discrete, akin to the genetically related spectrum of seemingly disparate neurodevelopmental disorders. $^{34,92}$ Combining clinically distinct CHD (e.g., multiple conotruncal defects) can increase power for rare variant identification and analyses. ${ }^{39,53,57}$ Common and rare genetic modifiers of expression remain to be discovered (Box 1).

\section{FUTURE CONSIDERATIONS}

Individually rare CNVs-gains and losses, inherited and de novoare collectively important genetic factors contributing to abnormal heart and great vessel development in humans. All rare variants sufficiently studied to date are characterised by some degree of variable cardiac and extra-cardiac expression. Many also display reduced penetrance. Estimating these parameters is challenging, and can be confounded by ascertainment bias and reproductive fitness effects. ${ }^{19,67,89,90}$ The individual rarity of nonrecurrent CNVs has meant that there is often insufficient evidence (be it epidemiology, cell biology or model organism-based) to conclude causality. Large human sample sizes including the wealth of data generated from clinical use of CMA, high-throughput model organism screening, ${ }^{106}$ and targeted mutagenesis using CRISPR/Cas9 technology will all help in this endeavour.

Epistatic interactions are not well studied in human CHD. Our ability to provide increasingly personalised counselling will be determined by our understanding of modifiers of expression. Elegant experiments in mice with heterozygous $N k \times 2-5$ mutations demonstrate the layers of complexity that we can expect to encounter even in individuals with pathogenic single-gene mutations. $^{107,108}$ For CNV, genome-wide molecular studies in cohorts with $22 q 11.2$ deletions provide the template for studying other recurrent variants (Box 1). For example, an immediate next step for many CNVs would be to carefully define the breakpoints and study the intact allele (Figure 2). The success of this approach is best illustrated by distal 1q21.1 deletions causing TAR syndrome. ${ }^{109}$

In our experience, the primary hope of many families and clinicians is that prenatal or early postnatal genetic, e.g., CMA, testing will help in predicting neurodevelopmental outcomes. This has not been the focus of most studies reviewed herein. However, research on CNV in CHD has been occurring in parallel with studies of developmental delay/intellectual disability, autism spectrum disorder and schizophrenia. ${ }^{33-36}$ Our understanding of genome-wide CNV, in terms of both general mechanisms (Figure 2) and specific loci, has been heavily influenced by neurodevelopmental disorders. A component of neurocognitive outcomes in individuals with CHD is genetically determined, and this can be dissected using the same approaches as studies of developmental disorders more generally. The expectation is that the frequency of VUS will decrease and the helpfulness of pathogenic CNV diagnoses will improve as more data accrue. The escalating uptake of methods that facilitate prenatal detection of CNV, including non-invasive prenatal testing that now permits screening for selected recurrent microdeletions, ${ }^{110}$ will further stoke demands for reliable prognostication and genetic counselling.

There is an impetus to consider in a similar fashion to CNV and structural variation the contribution of rare sequence-based changes to the genetic architecture of CHD. New genetic 
technologies have proved useful in advancing our understanding of CHD aetiopathogenesis, and increasing clinical diagnostic yield. Recently, whole-exome sequencing has begun to facilitate novel gene discovery. ${ }^{78,111}$ In the two largest whole-exome sequencing study to date, Zaidi et al. and Homsy et al. demonstrated enrichment of de novo point mutations in individuals with severe CHD and identified multiple new candidate genes. ${ }^{78,111}$ Interpretation of rare sequence variants will be informed by genes and pathways identified through rare CNV. WGS is the next logical step in this progression. The ability to comprehensively assess sequence and structural changes in coding and noncoding sequence genome-wide within an individual with CHD will undoubtedly identify new mechanisms that govern human cardiac morphogenesis. WGS offers the promise of improved resolution with respect to structural variation compared with CMA. The path forward involves the application of WGS on a large-scale. This will need to be married with careful phenotyping, family studies, and other hallmarks of excellence in genetic discovery study design. Personal WGS as a single universal genetic test in CHD that is cost-effective and of broadly applicable clinical utility is a highly anticipated but future consideration. ${ }^{112}$

\section{ACKNOWLEDGEMENTS}

ASB holds the Canada Research Chair in Schizophrenia Genetics and Genomic Disorders, and the Dalglish Chair in 22q11.2 Deletion Syndrome. This work was supported by the University of Toronto McLaughlin Centre and Canadian Institutes of Health Research (MOP 97800). The authors thank Gladys Wong and Sean Bekeschus for assistance with manuscript preparation.

\section{COMPETING INTERESTS}

The authors declare no conflict of interest.

\section{REFERENCES}

1. Hoffman, J. I. \& Kaplan, S. The incidence of congenital heart disease. J. Am. Coll. Cardiol. 39, 1890-1900 (2002).

2. van der Linde, D. et al. Birth prevalence of congenital heart disease worldwide: a systematic review and meta-analysis. J. Am. Coll. Cardiol. 58, 2241-2247 (2011).

3. van der Bom, T. et al. The changing epidemiology of congenital heart disease. Nat. Rev. Cardiol. 8, 50-60 (2011).

4. Zhang, Y. F., Zeng, X. L., Zhao, E. F. \& Lu, H. W. Diagnostic value of fetal echocardiography for congenital heart disease: a systematic review and meta-analysis. Medicine (Baltimore) 94, e1759 (2015).

5. Friedberg, M. K. et al. Prenatal detection of congenital heart disease. J. Pediatr. 155, 26-31, 31.e1 (2009).

6. Bahtiyar, M. O. \& Copel, J. A. Screening for congenital heart disease during anatomical survey ultrasonography. Obstet. Gynecol. Clin. North Am. 42, 209-223 (2015).

7. Donofrio, M. T. et al. Diagnosis and treatment of fetal cardiac disease: a scientific statement from the American Heart Association. Circulation 129, 2183-2242 (2014).

8. Holland, B. J., Myers, J. A. \& Woods, C. R. Jr. Prenatal diagnosis of critical congenital heart disease reduces risk of death from cardiovascular compromise prior to planned neonatal cardiac surgery: a meta-analysis. Ultrasound Obstet. Gynecol. 45, 631-638 (2015).

9. Khoshnood, B. et al. Trends in prenatal diagnosis, pregnancy termination, and perinatal mortality of newborns with congenital heart disease in France, 1983-2000: a population-based evaluation. Pediatrics 115, 95-101 (2005).

10. Silversides, C. K. et al. Canadian Cardiovascular Society 2009 Consensus Conference on the management of adults with congenital heart disease: shunt lesions. Can. J. Cardiol. 26, e70-e79 (2010).

11. Silversides, C. K. et al. Canadian Cardiovascular Society 2009 Consensus Conference on the management of adults with congenital heart disease: outflow tract obstruction, coarctation of the aorta, tetralogy of Fallot, Ebstein anomaly and Marfan's syndrome. Can. J. Cardiol. 26, e80-e97 (2010).

12. Silversides, C. K. et al. Canadian Cardiovascular Society 2009 Consensus Conference on the management of adults with congenital heart disease: complex congenital cardiac lesions. Can. J. Cardiol. 26, e98-117 (2010).

13. Bhatt, A. B. et al. Congenital heart disease in the older adult: a scientific statement from the American Heart Association. Circulation 131, 1884-1931 (2015).

14. Munoz-Chapuli, R. \& Perez-Pomares, J. M. Cardiogenesis: an embryological perspective. J. Cardiovasc. Transl. Res. 3, 37-48 (2010).
15. Egbe, A., Uppu, S., Lee, S., Ho, D. \& Srivastava, S. Prevalence of associated extracardiac malformations in the congenital heart disease population. Pediatr. Cardiol. 35, 1239-1245 (2014).

16. Marino, B. S. et al. Neurodevelopmental outcomes in children with congenital heart disease: evaluation and management: a scientific statement from the American Heart Association. Circulation 126, 1143-1172 (2012).

17. Marelli, A. J., Mackie, A. S., lonescu-lttu, R., Rahme, E. \& Pilote, L. Congenital heart disease in the general population: changing prevalence and age distribution. Circulation 115, 163-172 (2007).

18. Oyen, N. et al. Recurrence of congenital heart defects in families. Circulation 120, 295-301 (2009).

19. Chin-Yee, N. J., Costain, G., Swaby, J. A., Silversides, C. K. \& Bassett, A. S. Reproductive fitness and genetic transmission of tetralogy of Fallot in the molecular age. Circ. Cardiovasc. Genet. 7, 102-109 (2014).

20. Pierpont, M. E. et al. Genetic basis for congenital heart defects: current knowledge: a scientific statement from the American Heart Association Congenital Cardiac Defects Committee, Council on Cardiovascular Disease in the Young: endorsed by the American Academy of Pediatrics. Circulation 115, 3015-3038 (2007).

21. Gill, H. K., Splitt, M., Sharland, G. K. \& Simpson, J. M. Patterns of recurrence of congenital heart disease: an analysis of 6,640 consecutive pregnancies evaluated by detailed fetal echocardiography. J. Am. Coll. Cardiol. 42, 923-929 (2003).

22. Jenkins, K. J. et al. Noninherited risk factors and congenital cardiovascular defects: current knowledge: a scientific statement from the American Heart Association Council on Cardiovascular Disease in the Young: endorsed by the American Academy of Pediatrics. Circulation 115, 2995-3014 (2007).

23. Patel, S. S. \& Burns, T. L. Nongenetic risk factors and congenital heart defects. Pediatr. Cardiol. 34, 1535-1555 (2013).

24. Nora, J. J. Multifactorial inheritance hypothesis for the etiology of congenital heart diseases. The genetic-environmental interaction. Circulation 38, 604-617 (1968).

25. Edwards, J. J. \& Gelb, B. D. Genetics of congenital heart disease. Curr. Opin. Cardiol. 31, 235-241 (2016).

26. Wimalasundera, R. C. \& Gardiner, H. M. Congenital heart disease and aneuploidy. Prenat. Diagn. 24, 1116-1122 (2004).

27. van Karnebeek, C. D. \& Hennekam, R. C. Associations between chromosomal anomalies and congenital heart defects: a database search. Am. J. Med. Genet. 84, 158-166 (1999).

28. Pober, B. R. Williams-Beuren syndrome. N. Engl. J. Med. 362, 239-252 (2010).

29. McDonald-McGinn, D. M. et al. 22q11.2 deletion syndrome. Nat. Rev. Dis. Prim. 1, 15071 (2015).

30. Scambler, P. J. 22q11 deletion syndrome: a role for TBX1 in pharyngeal and cardiovascular development. Pediatr. Cardiol. 31, 378-390 (2010).

31. lafrate, A. J. et al. Detection of large-scale variation in the human genome. Nat. Genet. 36, 949-951 (2004).

32. Sebat, J. et al. Large-scale copy number polymorphism in the human genome. Science 305, 525-528 (2004).

33. Lee, C. \& Scherer, S. W. The clinical context of copy number variation in the human genome. Expert Rev. Mol. Med. 12, e8 (2010).

34. Cooper, G. M. et al. A copy number variation morbidity map of developmental delay. Nat. Genet. 43, 838-846 (2011).

35. Miller, D. T. et al. Consensus statement: chromosomal microarray is a first-tier clinical diagnostic test for individuals with developmental disabilities or congenital anomalies. Am. J. Hum. Genet. 86, 749-764 (2010).

36. Costain, G. et al. Pathogenic rare copy number variants in community-based schizophrenia suggest a potential role for clinical microarrays. Hum. Mol. Genet. 22, 4485-4501 (2013).

37. Thienpont, B. et al. Submicroscopic chromosomal imbalances detected by array-CGH are a frequent cause of congenital heart defects in selected patients. Eur. Heart J. 28, 2778-2784 (2007).

38. Silversides, C. K. et al. Rare copy number variations in adults with tetralogy of Fallot implicate novel risk gene pathways. PLoS Genet. 8, e1002843 (2012).

39. Soemedi, R. et al. Contribution of global rare copy-number variants to the risk of sporadic congenital heart disease. Am. J. Hum. Genet. 91, 489-501 (2012).

40. Hitz, M. P. et al. Rare copy number variants contribute to congenital left-sided heart disease. PLoS Genet. 8, e1002903 (2012).

41. Lalani, S. R. et al. Rare DNA copy number variants in cardiovascular malformations with extracardiac abnormalities. Eur. J. Hum. Genet. 21, 173-181 (2013).

42. Xie, L. et al. Rare de novo copy number variants in patients with congenital pulmonary atresia. PLOS ONE 9, e96471 (2014).

43. Zhao, W. et al. High-resolution analysis of copy number variants in adults with simple-to-moderate congenital heart disease. Am. J. Med. Genet. A 161A, 3087-3094 (2013) 
44. White, P. S. et al. Analysis of chromosomal structural variation in patients with congenital left-sided cardiac lesions. Birth Defects Res. A Clin. Mol. Teratol. 100, 951-964 (2014).

45. Greenway, S. C. et al. De novo copy number variants identify new genes and loci in isolated sporadic tetralogy of Fallot. Nat. Genet. 41, 931-935 (2009).

46. Priest, J. R. et al. Rare copy number variants in isolated sporadic and syndromic atrioventricular septal defects. Am. J. Med. Genet. A 158A, 1279-1284 (2012).

47. Warburton, D. et al. The contribution of de novo and rare inherited copy number changes to congenital heart disease in an unselected sample of children with conotruncal defects or hypoplastic left heart disease. Hum. Genet. 133, 11-27 (2014).

48. Fakhro, K. A. et al. Rare copy number variations in congenital heart disease patients identify unique genes in left-right patterning. Proc. Natl Acad. Sci. USA 108, 2915-2920 (2011).

49. Goldmuntz, E. et al. Microdeletions and microduplications in patients with congenital heart disease and multiple congenital anomalies. Congenit. Heart Dis. 6, 592-602 (2011).

50. Tomita-Mitchell, A. et al. Human gene copy number spectra analysis in congenital heart malformations. Physiol. Genomics 44, 518-541 (2012).

51. Breckpot, J. et al. Challenges of interpreting copy number variation in syndromic and non-syndromic congenital heart defects. Cytogenet. Genome Res. 135, 251-259 (2011).

52. Thorsson, T. et al. Chromosomal imbalances in patients with congenital cardiac defects: a meta-analysis reveals novel potential critical regions involved in heart development. Congenit. Heart Dis. 10, 193-208 (2015).

53. Costain, G. et al. Genome-wide rare copy number variations contribute to genetic risk for transposition of the great arteries. Int. J. Cardiol. 204, 115-121 (2016).

54. Kim, D. S. et al. Burden of potentially pathologic copy number variants is higher in children with isolated congenital heart disease and significantly impairs covariate-adjusted transplant-free survival. J. Thorac. Cardiovasc. Surg. 151, 1147-1151.e4 (2016),

55. Sanchez-Castro, M. et al. Search for rare copy-number variants in congenital heart defects identifies novel candidate genes and a potential role for FOXC1 in patients with coarctation of the aorta. Circ. Cardiovasc. Genet. 9, 86-94 (2016).

56. An, Y. et al. Genome-wide copy number variant analysis for congenital ventricular septal defects in Chinese Han population. BMC Med. Genomics 9, 2 (2016).

57. Glessner, J. T. et al. Increased frequency of de novo copy number variants in congenital heart disease by integrative analysis of single nucleotide polymorphism array and exome sequence data. Circ. Res. 115, 884-896 (2014).

58. Carey, A. S. et al. Effect of copy number variants on outcomes for infants with single ventricle heart defects. Circ. Cardiovasc. Genet. 6, 444-451 (2013).

59. Peyvandi, S. et al. 22q11.2 deletions in patients with conotruncal defects: data from 1,610 consecutive cases. Pediatr. Cardiol. 34, 1687-1694 (2013).

60. Osoegawa, K. et al. Identification of novel candidate gene loci and increased sex chromosome aneuploidy among infants with conotruncal heart defects. Am. J. Med. Genet. A 164A, 397-406 (2014).

61. Fung, W. L. et al. Practical guidelines for managing adults with $22 q 11.2$ deletion syndrome. Genet. Med. 17, 599-609 (2015).

62. Bassett, A. S. et al. Practical guidelines for managing patients with $22 q 11.2$ deletion syndrome. J. Pediat. 159, 332-339 (2011).

63. Bassett, A. S. et al. Clinical features of 78 adults with $22 q 11$ deletion syndrome. Am. J. Med. Genet. A 138, 307-313 (2005).

64. O'Byrne, M. L. et al. 22q11.2 Deletion syndrome is associated with increased perioperative events and more complicated postoperative course in infants undergoing infant operative correction of truncus arteriosus communis or interrupted aortic arch. J. Thorac. Cardiovasc. Surg. 148, 1597-1605 (2014).

65. Portnoi, M. F. Microduplication 22q11.2: a new chromosomal syndrome. Eur. J. Med. Genet. 52, 88-93 (2009).

66. Christiansen, J. et al. Chromosome 1q21.1 contiguous gene deletion is associated with congenital heart disease. Circ. Res. 94, 1429-1435 (2004).

67. Dolcetti, A. et al. 1q21.1 Microduplication expression in adults. Genet. Med. 15, 282-289 (2013).

68. Soemedi, R. et al. Phenotype-specific effect of chromosome 1q21.1 rearrangements and GJA5 duplications in 2436 congenital heart disease patients and 6760 controls. Hum. Mol. Genet. 21, 1513-1520 (2012).

69. Verhagen, J. M. et al. Phenotypic variability associated with a large recurrent 1q21.1 microduplication in a three-generation family. Mol. Syndromol. 6, 71-76 (2015).

70. Chaldoupi, S. M., Loh, P., Hauer, R. N., de Bakker, J. M. \& van Rijen, H. V. The role of connexin40 in atrial fibrillation. Cardiovasc. Res. 84, 15-23 (2009).

71. Garg, V. et al. GATA4 mutations cause human congenital heart defects and reveal an interaction with TBX5. Nature 424, 443-447 (2003).
72. Pehlivan, T. et al. GATA4 haploinsufficiency in patients with interstitial deletion of chromosome region 8p23.1 and congenital heart disease. Am. J. Med. Genet. 83, 201-206 (1999).

73. Wat, J. J. \& Wat, M. J. Sox7 in vascular development: review, insights and potential mechanisms. Int. J. Dev. Biol. 58, 1-8 (2014).

74. Vanlerberghe, C. et al. 15q11.2 microdeletion (BP1-BP2) and developmental delay, behaviour issues, epilepsy and congenital heart disease: a series of 52 patients. Eur. J. Med. Genet. 58, 140-147 (2015).

75. Cafferkey, M., Ahn, J. W., Flinter, F. \& Ogilvie, C. Phenotypic features in patients with 15q11.2(BP1-BP2) deletion: further delineation of an emerging syndrome. Am. J. Med. Genet. A 164A, 1916-1922 (2014).

76. Hashemi, B. et al. Deletion of 15q11.2(BP1-BP2) region: Further evidence for lack of phenotypic specificity in a pediatric population. Am. J. Med. Genet. A 167A, 2098-2102 (2015).

77. Costain, G., Roche, S. L., Scherer, S. W., Silversides, C. K. \& Bassett, A. S. Rare copy number variations in an adult with transposition of the great arteries emphasize the importance of updated genetic assessments in syndromic congenital cardiac disease. Int. J. Cardiol. 203, 516-518 (2016).

78. Homsy, J. et al. De novo mutations in congenital heart disease with neurodevelopmental and other congenital anomalies. Science 350, 1262-1266 (2015).

79. Merico, D. et al. MicroRNA dysregulation, gene networks, and risk for schizophrenia in 22q11.2 deletion syndrome. Front. Neurol. 5, 238 (2014).

80. Xing, H. J. et al. Identification of microRNAs present in congenital heart disease associated copy number variants. Eur. Rev. Med. Pharmacol. Sci. 17, 2114-2120 (2013).

81. Jansen, F. A. et al. Array comparative genomic hybridization and fetal congenital heart defects: a systematic review and meta-analysis. Ultrasound Obstet. Gynecol. 45, 27-35 (2015)

82. Shaffer, L. G. et al. Experience with microarray-based comparative genomic hybridization for prenatal diagnosis in over 5000 pregnancies. Prenat. Diagn. 32, 976-985 (2012).

83. Geng, J. et al. Chromosome microarray testing for patients with congenital heart defects reveals novel disease causing loci and high diagnostic yield. BMC Genomics 15, 1127 (2014).

84. Connor, J. A. et al. Genetic testing practices in infants with congenital heart disease. Congenit. Heart Dis. 9, 158-167 (2014).

85. Buckley, J. R., Kavarana, M. N., Chowdhury, S. M. \& Scheurer, M. A. Current practice and utility of chromosome microarray analysis in infants undergoing cardiac surgery. Congenit. Heart Dis. 10, E131-E138 (2015).

86. Helm, B. M. \& Freeze, S. L. Genetic evaluation and use of chromosome microarray in patients with isolated heart defects: benefits and challenges of a new model in cardiovascular care. Front. Cardiovasc. Med. 3, 19 (2016).

87. Chaix, M. A., Andelfinger, G. \& Khairy, P. Genetic testing in congenital heart disease: a clinical approach. World J. Cardiol. 8, 180-191 (2016).

88. van Engelen, K. et al. The value of the clinical geneticist caring for adults with congenital heart disease: diagnostic yield and patients' perspective. Am. J. Med. Genet. A 161A, 1628-1637 (2013).

89. Costain, G., Chow, E. W., Silversides, C. K. \& Bassett, A. S. Sex differences in reproductive fitness contribute to preferential maternal transmission of 22 q11.2 deletions. J. Med. Genet. 48, 819-824 (2011).

90. Costain, G. Parental expression is overvalued in the interpretation of rare inherited variants. Eur. J. Hum. Genet. 23, 4-7 (2015).

91. Hinton, R. B. Jr. et al. Hypoplastic left heart syndrome is heritable. J. Am. Coll. Cardiol. 50, 1590-1595 (2007).

92. Bassett, A. S., Scherer, S. W. \& Brzustowicz, L. M. Copy number variations in schizophrenia: critical review and new perspectives on concepts of genetics and disease. Am. J. Psychiatry 167, 899-914 (2010).

93. Michielon, G. et al. Genetic syndromes and outcome after surgical correction of tetralogy of Fallot. Ann. Thorac. Surg. 81, 968-975 (2006).

94. Anaclerio, S. et al. Conotruncal heart defects: impact of genetic syndromes on immediate operative mortality. Ital. Heart J. 5, 624-628 (2004).

95. Gaynor, J. W. et al. Patient characteristics are important determinants of neurodevelopmental outcome at one year of age after neonatal and infant cardiac surgery. J. Thorac. Cardiovasc. Surg. 133, 1344-1353, 1353.e1-3 (2007).

96. Costain, G. et al. 13q13.1-q13.2 deletion in tetralogy of Fallot: clinical report and a literature review. Int. J. Cardiol. 146, 134-139 (2011).

97. Girirajan, S. et al. Refinement and discovery of new hotspots of copy-number variation associated with autism spectrum disorder. Am. J. Hum. Genet. 92, 221-237 (2013).

98. Stefansson, H. et al. CNVs conferring risk of autism or schizophrenia affect cognition in controls. Nature 505, 361-366 (2014).

99. Preuss, C. \& Andelfinger, G. Genetics of heart failure in congenital heart disease. Can. J. Cardiol. 29, 803-810 (2013).

100. Bassett, A. S. et al. Premature death in adults with 22 q11.2 deletion syndrome. J. Med. Genet. 46, 324-330 (2009). 
101. Arndt, A. K. et al. Fine mapping of the $1 \mathrm{p} 36$ deletion syndrome identifies mutation of PRDM16 as a cause of cardiomyopathy. Am. J. Hum. Genet. 93, 67-77 (2013).

102. de Leeuw, N. \& Houge, G. Loss of PRDM16 is unlikely to cause cardiomyopathy in 1 p36 deletion syndrome. Am. J. Hum. Genet. 94, 153-154 (2014).

103. Arndt, A. K., Macrae, C. A. \& Klaassen, S. Reponse to De Leeuw and Houge. Am. J. Hum. Genet. 94, 154-155 (2014).

104. Jordan, V. K., Zaveri, H. P. \& Scott, D. A. 1 p36 deletion syndrome: an update. Appl. Clin. Genet. 8, 189-200 (2015).

105. Fregeau, B. et al. De novo mutations of RERE cause a genetic syndrome with features that overlap those associated with proximal 1p36 deletions. Am. J. Hum. Genet. 98, 963-970 (2016).

106. Li, Y. et al. Global genetic analysis in mice unveils central role for cilia in congenital heart disease. Nature 521, 520-524 (2015).

107. Winston, J. B. et al. Heterogeneity of genetic modifiers ensures normal cardiac development. Circulation 121, 1313-1321 (2010).

108. Winston, J. B. et al. Complex trait analysis of ventricular septal defects caused by Nkx2-5 mutation. Circ. Cardiovasc. Genet. 5, 293-300 (2012).

109. Albers, C. A. et al. Compound inheritance of a low-frequency regulatory SNP and a rare null mutation in exon-junction complex subunit RBM8A causes TAR syndrome. Nat. Genet. 44, 435-439, S1-2 (2012).

110. Benn, P. Expanding non-invasive prenatal testing beyond chromosomes 21, 18, 13, X and Y. Clin. Genet. doi:10.1111/cge.12818 (2016).

111. Zaidi, S. et al. De novo mutations in histone-modifying genes in congenital heart disease. Nature 498, 220-223 (2013).

112. Stavropoulos, D. J. et al. Whole-genome sequencing expands diagnostic utility and improves clinical management in paediatric medicine. NPJ Gen. Med. 1, 15012 (2016).

113. Mlynarski, E. E. et al. Copy-number variation of the glucose transporter Gene SLC2A3 and congenital heart defects in the 22q11.2 deletion syndrome. Am. J. Hum. Genet. 96, 753-764 (2015).
114. Racedo, S. E. et al. Mouse and human CRKL is dosage sensitive for cardiac outflow tract formation. Am. J. Hum. Genet. 96, 235-244 (2015).

115. Chapnik, E., Sasson, V., Blelloch, R. \& Hornstein, E. Dgcr8 controls neural crest cells survival in cardiovascular development. Dev. Biol. 362, 50-56 (2012).

116. Guo, T. et al. Genotype and cardiovascular phenotype correlations with TBX1 in 1,022 Velo-Cardio-Facial/DiGeorge/22q11.2 Deletion Syndrome patients. Hum. Mutat. 32, 1278-1289 (2011).

117. Swaby, J. A. et al. Complex congenital heart disease in unaffected relatives of adults with 22q11.2 deletion syndrome. Am. J. Cardiol. 107, 466-471 (2011).

118. Digilio, M. C. et al. Familial recurrence of nonsyndromic congenital heart defects in first degree relatives of patients with deletion 22q11.2. Am. J. Med. Genet. 134 A, 158-164 (2005).

119. Mlynarski, E. E. et al. Rare copy number variants and congenital heart defects in the 22q11.2 deletion syndrome. Hum. Genet. 135, 273-285 (2016).

120. Guo, T. et al. Histone modifier genes alter conotruncal heart phenotypes in 22q11.2 deletion syndrome. Am. J. Hum. Genet. 97, 869-877 (2015).

121. Kearney, H. M. et al. American College of Medical Genetics standards and guidelines for interpretation and reporting of postnatal constitutional copy number variants. Genet. Med. 13, 680-685 (2011).

This work is licensed under a Creative Commons Attribution 4.0 International License. The images or other third party material in this article are included in the article's Creative Commons license, unless indicated otherwise in the credit line; if the material is not included under the Creative Commons license, users will need to obtain permission from the license holder to reproduce the material. To view a copy of this license, visit http://creativecommons.org/licenses/ by/4.0/

(c) The Author(s) 2016 